\title{
Mythos
}

Rivista di Storia delle Religioni

$13 \mid 2019$

Varia

\section{Una nota sul dionisiaco di Furio Jesi: inattuale e originale}

A Comment on Furio Jesi's Dionysian: Untimely and Original

\section{Francesco Massa}

\section{(2) OpenEdition \\ Journals}

Edizione digitale

URL: http://journals.openedition.org/mythos/843

ISSN: 2037-7746

\section{Editore}

Salvatore Sciascia Editore

\section{Notizia bibliografica digitale}

Francesco Massa, « Una nota sul dionisiaco di Furio Jesi: inattuale e originale », Mythos [Online], 13 |

2019, online dal 01 décembre 2019, consultato il 26 décembre 2019. URL : http://

journals.openedition.org/mythos/843

Questo documento è stato generato automaticamente il 26 dicembre 2019.

Mythos 


\title{
Una nota sul dionisiaco di Furio Jesi: inattuale e originale
}

\author{
A Comment on Furio Jesi's Dionysian: Untimely and Original
}

\author{
Francesco Massa
}

Non ritornano i sassi e le selve.

Ci sono. Quel che è stato sarà.

Cesare Pavese, Dialoghi con Leucò ${ }^{1}$

1 Nel 1972, Furio Jesi scrive la prefazione alla traduzione italiana del libro di Henri Jeanmaire, Dionysos. Histoire du culte de Bacchus, che era stato pubblicato a Parigi una ventina di anni prima, nel $1951^{2}$. Intitolato Inattualità di Dioniso, lo scritto analizza alcuni aspetti del dionisismo dall'antichità al tempo presente. È un saggio denso, ricco di riferimenti letterari e filosofici, in linea con la scrittura di Jesi, in cui l'autore riprende e sviluppa alcuni temi centrali nel suo pensiero, dal ruolo del mito al rapporto tra antico e contemporaneità. In occasione della pubblicazione di questa traduzione, Jesi scrive anche un'appendice su Nuovi documenti e nuovi studi sulle origini di Dioniso, che tiene conto del cambiamento di paradigma negli studi dionisiaci in seguito alla decifrazione della lineare B, avvenuta dopo la pubblicazione dell'edizione originale del libro di Jeanmaire ${ }^{3}$. Questi due testi rappresentano un binomio perfetto degli interessi di Jesi. Se nella prefazione propone un saggio centrato più sul dionisiaco che su Dioniso, nell'appendice Jesi si serve della sua formazione filologica per analizzare con acribia le nuove scoperte provenienti dall'età micenea. Le poche pagine che seguono si propongono di situare il testo di Jesi nel panorama degli studi dionisiaci e di metterne in evidenza gli elementi di attualità.

\section{Il rinnovamento degli studi su Dioniso e la traduzione del volume di Henri Jeanmaire}

2 Gli anni compresi tra la data dell'uscita dell'opera di Jeanmaire e quella della traduzione italiana segnano una svolta nella storia degli studi dionisiaci novecenteschi. L'anno chiave di è proprio il 1951 quando, oltre al Dionysos di Jeanmaire, viene 
pubblicato il lavoro più significativo di Eric R. Dodds, The Greeks and the Irrational: il volume conteneva in appendice un saggio sul menadismo che per la prima volta proponeva un'interpretazione della mania dionisiaca attraverso la comparazione con culture e fenomeni moderni, insistendo soprattutto sulla dimensione "psicologica" del culto bacchico". I lavori di Dodds e Jeanmaire impressero un nuovo corso allo studio della figura di Dioniso, influenzando sensibilmente la critica dei decenni successivi. Si potrebbe forse aggiungere a questi due volumi il libro di Adrien Bruhl dedicato alla storia del Liber romano, pubblicato a Parigi, due anni più tardi, nel 1953, sebbene il suo approccio sia sicuramente più tradizionale ${ }^{5}$.

3 Un elemento accomuna la maggior parte degli studi di quegli anni, probabilmente con la sola eccezione di Dodds: la ricerca delle origini del culto di Dioniso e dalla ricostruzione delle sue radici geografiche ${ }^{6}$. Il dio era considerato di origine non greca, secondo una teoria già esistente nell'antichità e poi confermata dai primi studi moderni, e questa origine straniera era considerata essenziale per comprendere le sfere di influenza del dio e soprattutto i suoi aspetti più barbari rispetto agli ideali della classicità. Nei primi anni dell'Ottocento, Friedrich Creuzer scrive quella che può essere considerata la prima monografia sul dio, in cui Dioniso era rappresentato come una divinità non greca, probabilmente di origine egiziana ${ }^{7}$. Nella seconda metà del XIX secolo, Erwin Rohde confermò la provenienza straniera del dio, proponendone un'origine dalla Tracia ${ }^{8}$. Nei decenni tra la fine del XIX e l'inizio del XX secolo, la questione delle origini della cultura greca e, di conseguenza, delle divinità elleniche era al centro degli interessi degli studiosi di antichità classica. In questo panorama, gli studi dionisiaci offrivano un terreno fertile per applicare queste ricerche, non soltanto per quanto riguarda lo studio dell'origine geografica del dio, ma anche per le ipotesi sull'origine del teatro e, in particolare, della tragedia ateniese. È come se gli studiosi di quest'epoca pensassero che la "ricerca delle origini" offrisse la chiave per interpretare l'“essenza" del dionisismo e, più in generale, della cultura greca'. Gli studi sulle religioni antiche si situavano quindi in quello che Marc Bloch aveva definito "l'idolo delle origini”, un campo che toccava una grande parte della storiografia dell'epoca ${ }^{10}$.

4 Tra il 1952 e il 1953, immediatamente dopo la pubblicazione dei volumi di Jeanmaire e di Dodds, un altro studio fondamentale contribuì a far ripensare la questione delle origini di Dioniso e del suo rapporto con la cultura greca. In quell'anno, infatti, Michael Ventris e John Chadwick proposero la loro teoria sull'origine greca della lineare B e qualche anno più tardi gli archeologi ritrovarono il nome di Dioniso su alcune tavolette micenee provenienti da Pilo: la scoperta portò a una decostruzione delle teorie sull'estraneità di Dioniso, poiché il dio non era stato successivamente importato da una terra straniera. Dioniso era in tutto e per tutto un dio greco ${ }^{11}$.

5 Infine, non si può dimenticare il ruolo giocato nella storiografia degli anni Cinquanta e Sessanta dagli studi su Dioniso di Károly Kerényi: sebbene il volume Dionysos sia stato pubblicato postumo nel 1976, e quindi dopo l'uscita della traduzione italiana del libro di Jeanmaire, lo studioso aveva già pubblicato nel 1951, a seguito di una conferenza svoltasi a Roma, un importante saggio sul sacrificio dionisiaco in cui anticipava alcuni degli elementi che sarebbero poi confluiti della sua monografia dionisiaca, come la concezione di un culto del vino mediterraneo che poteva aver costituito un sostrato comune alla base del culto di Dioniso, dell'orfismo e del cristianesimo ${ }^{12}$.

6 È in questo vivace contesto di rinnovamento metodologico e filologico che viene pubblicata la traduzione italiana del volume di Jeanmaire, nella "Nuova Biblioteca 
scientifica Einaudi", nata nel 1965 riprendendo i temi di due collane che avevano cessato la loro attività, "Biblioteca di cultura scientifica ed economica" e "Collezione di studi religiosi, etnologici e psicologici". L'obiettivo era quello di "promuovere interventi di alto livello culturale nell'ambito di diverse discipline"13. La "Collezione di studi religiosi, etnologici e psicologici" (la cosiddetta "Collana viola") era stata fondata da Ernesto De Martino e Cesare Pavese nel 1948 e aveva pubblicato ventisette volumi fino al $1955^{14}$. Nonostante la volontà esplicitata di proseguire nella "Nuova biblioteca scientifica" le pubblicazioni della "Collana viola", è lecito interrogarsi sulla scelta di includere il testo di Jeanmaire in questa collana. Si tratta, in effetti, dell'unico volume dedicato al mondo antico e anche l'unico volume dedicato a una tematica religiosa. I titoli della collana si concentrano soprattutto su temi di economia, scienze politiche e filosofia politica. Soltanto due volumi affrontano tematiche di antropologia: quello di Marcel Mauss e quello di Ernesto de Martino ${ }^{15}$. Non stupisce quindi che Jesi abbia accettato di partecipare a questo progetto culturale einaudiano ${ }^{16}$.

7 L'approccio originale di Jeanmaire doveva aver attirato l'attenzione di Jesi, in particolare per quanto riguardava l'uso innovativo dei lavori degli etnografi, degli antropologi e anche degli psichiatri che erano serviti allo studioso francese per spiegare i fenomeni dionisiaci e, in particolare, il menadismo. La prefazione di Jesi, tuttavia, non vuole essere uno studio storico sul dio greco Dioniso, bensì un saggio sulla categoria del "dionisiaco" nella storia culturale europea. È un progetto ambizioso che tocca alcuni dei temi più significativi che tradizionalmente erano stati associati a Dioniso. In questo contesto, il Dioniso di Jesi è, prima di tutto, il dio dell'enthousiasmos, della mania, della possessione e dell'iniziazione: lo studioso si situa dunque nel solco degli studi su Dioniso successivi al 1951.

Tuttavia quello che interessa Jesi è il ruolo di Dioniso nella storia del pensiero occidentale moderno e, soprattutto, nei pensatori tedeschi. Leggendo le pagine della prefazione si ha la netta sensazione che, pur traendo origine dall'analisi delle fonti antiche, il testo di Jesi sia tutto volto al presente. È per questo motivo che l'“inattualità" di Dioniso deve essere intesa chiaramente in senso nietzschiano, come Unzeitgemäß, vale a dire come una dimensione che va contro alle condizioni attuali. Il dionisiaco diviene lo strumento per scardinare lo stato presente delle cose. Per quanto alcuni temi si richiamino nei due testi, il lettore può facilmente notare lo scarto che esiste tra lo stile, il contenuto e gli obiettivi della prefazione di Jesi e lo studio di Jeanmaire.

\section{Dioniso nelle stanze rinascimentali e l'eco di Pavese}

9 Il saggio di Jesi prende avvio evocando alcune immagini delle Stanze di Angelo Poliziano e della Canzona di Bacco di Lorenzo il Magnifico. I due testi mettono in scena l'incontro tra Dioniso e Arianna e rappresentano i due amanti divini come le manifestazioni della potenza di Venere ${ }^{17}$. In uno dei capitoli del suo volume sul mito, uscito nell'anno successivo alla pubblicazione della traduzione italiana del libro di Jeanmaire, Jesi definisce il periodo rinascimentale come l'ultimo momento in cui il mito visse in una forma autentica e genuina, rimanendo sostanzialmente estraneo alla "scienza del mito" 18. Il fatto che la prefazione di Jesi prenda avvio proprio con una riflessione sul Rinascimento non è casuale e dimostra l'originalità dell'approccio di Jesi: in questo incipit, lo studioso vuole evitare di riprendere le teorie sul dionisismo sviluppate dopo il XVIII secolo in Germania e poi soprattutto da Nietzsche. Cominciare dalla letteratura 
fiorentina di fine Quattrocento è l'occasione di riflettere sull'azione del mito e sulla sua sopravvivenza nelle epoche successive al mondo classico, uno dei temi fondamentali dell'opera di Jesi ${ }^{19}$. Nella concezione dello studioso, il tempo del mito è immobile e si situa in un tempo anteriore rispetto a quello della realtà umana. Tempo del mito $\mathrm{e}$ tempo degli esseri umani sono profondamente e irrimediabilmente separati:

Poiché il mito non è mai allegoria, emblema, del destino umano, ma genuino precedente di esso; precedente, realtà diversa per eccellenza poiché precede la realtà umana. Nulla ci consente di credere che i devoti di Dioniso identificassero la sorte della loro "anima" con quella di Arianna, colpevole, uccisa, e poi risorta e glorificata per opera del dio. Possiamo ritenere, invece, che la prima parte dolorosa e colpevole della vicenda di Arianna coincidesse con un aspetto dell'effigie di Dioniso - quello oscuro -, mentre il trionfo della coppia dovrebbe corrispondere all'epifania dell'altro aspetto - quello luminoso ${ }^{20}$.

Numerosi aspetti di questo passo meritano di essere evidenziati. Prima di tutto, Jesi si oppone alla lettura del mito come semplice allegoria del destino umano. L'idea che il mito fosse soltanto un'allegoria, quasi un modo di parlare, è antica ed era diventata comune nell'interpretazione degli studiosi dell'ottocento ${ }^{21}$. Da questo punto di vista Jesi è prossimo alla concezione del mito espressa da Cesare Pavese nei Dialoghi con Leucò, composti nell'immediato dopoguerra e pubblicati nel 1947, su cui Jesi aveva avuto l'occasione di riflettere a lungo ${ }^{22}$. Secondo Pavese, il mito si compone di due dimensioni diverse e complementari: da un lato, il mito si produce nel momento dell'attività letteraria e soprattutto poetica, dall'altro appare in modo simbolico "come l'apprendimento di un bambino"23.

11 Su questa base, Jesi si interroga sul rapporto tra il dio e i suoi adepti, rifiutando che possa esistere una forma di assimilazione tra i devoti e Arianna, e rifiutando anche, più avanti, che si possa "intendere la sorte del dio come paradigma di quella dei devoti" ${ }^{24}$. Il motivo di questo rifiuto risiede proprio nell'interpretazione che lo studioso dà del mito: se è mediato dal mito, il rapporto dei devoti con il loro dio non può produrre una forma di imitazione; al contrario, esso è segnato dall'"abisso" che separa il mondo umano da quello divino. Diverso sarà invece, per Jesi, il cristianesimo che fonderà proprio sull'imitazione del dio-uomo la sua specificità. Dionisismo e cristianesimo sono dunque divergenti nella costruzione rituale che definisce il rapporto tra esseri umani e mondo divino, ma sono accomunati dalla funzione analoga dei due movimenti: "il rinnovamento di una visione dell'universo e del destino" 25 . Entrambi, secondo Jesi, fanno il loro ingresso nella storia attraverso una "violenta e iconoclasta distruzione dei valori tradizionali" ${ }^{26}$. Jesi riprende qui il parallelismo tra Dioniso e Cristo su cui aveva insistito soprattutto il Romanticismo tedesco, come vedremo nel paragrafo successivo.

Ritornando alla concezione del mito e del tempo ad esso associato, possiamo quindi concludere che per Jesi si tratta di un tempo lontano e un mondo che non è più accessibile agli uomini. L'eco dei Dialoghi con Leucò di Pavese diventa più intensa. Basti pensare alla figura di Prometeo, così come Pavese l'aveva rappresentata: non si trattava più dell'eroe romantico, fondatore e iniziatore dei saperi umani, ma del personaggio che segnava definitivamente la distanza tra il mondo degli dèi e degli uomini, colui che, solo, poteva fungere da intermediario poiché conservava un contatto con le divinità ${ }^{27}$. 


\section{Breve storia del dionisiaco tra antico e contemporaneo}

13 Jesi si sofferma poi sulla categoria del "dionisiaco" e sulla storia della sua interpretazione. La riflessione prende avvio dalla constatazione del ruolo di questa categoria nella cultura europea:

Riconoscere nel dionisiaco una costante dell'esperienza umana (o, se si vuole, in particolare un contenuto perenne di determinate forme di conoscenza) è una tentazione cui più volte si è consentito nella storia della cultura europea ${ }^{28}$.

14 Jesi era un fine conoscitore di questa storia della cultura europea, soprattutto tedesca, come sappiamo. Dagli ultimi decenni del XVIII secolo e poi soprattutto dall'inizio del XIX secolo, Dioniso e il suo mondo furono al centro di una riscoperta e di una riappropriazione da parte degli intellettuali del Romanticismo tedesco. La diffusione del dio fu senza precedenti. Non è un caso che si sia parlato, per questo periodo, di "epifania dionisiaca" ${ }^{2}$. La ricerca di una nuova mitologia guidava le riflessioni di personalità come Johann Gottfried Herder o Friedrich Wilhelm Schelling: costoro vedevano in Dioniso il "dio che viene", colui che avrebbe superato la civiltà moderna che non possedeva una mitologia propria ${ }^{30}$. Quasi nelle vesti di un nuovo messia, iniziatore di una rinascita religiosa, Dioniso era ovviamente associato a Cristo. ̇̀ sufficiente rinviare alle speculazioni letterarie e filosofiche di Novalis o di Friedrich Hölderlin, tra la fine del XVIII e l'inizio del XIX secolo ${ }^{31}$. Qualche anno più tardi, nel 1809, Friedrich Creuzer pubblicherà il suo già citato studio su Dioniso, in cui si affermava lo stretto legame tra cristianesimo e dionisismo ${ }^{32}$. Sono tutti autori che nutriranno in seguito le riflessioni di Nietzsche sul dionisiaco ${ }^{33}$. In questa prospettiva, Jesi doveva avere in mente anche la posizione di Kerényi che proponeva di leggere nelle divinità del mondo greco alcuni archetipi della psiche, sulla scia delle teorie junghiane ${ }^{34}$.

15 Jesi rifiuta questa interpretazione totalizzante del dionisiaco e mette in guardia il lettore da questa tentazione intellettuale, rifacendosi a quanto espresso da Jeanmaire:

Henri Jeanmaire in tutto il suo volume sfugge generalmente al paradosso della "perennità temporale" usufruendo di tecniche non dissimili da quelle della ricerca naturalistica, e cioè isolando fra le testimonianze del fenomeno antico certe relazioni meccaniche ripetibili e ripetute in contesti cronologicamente più vicini (se non addirittura, contemporanei a noi). [...] Di qui l'osservazione conclusiva di Jeanmaire, che può suonare sconcertante, sulla "inattualità" di Dioniso. Jeanmaire ha probabilmente ragione: Dioniso non è "attuale", e solo con arbitrio si può ravvisare nella religione dionisiaca storicamente configurata un "dionisismo" perenne. Ciò non significa, però, che Dioniso quale "dio del dolore" non abbia goduto di una fortuna secolare, largamente posteriore al limite storico della devozione organizzata verso di lui. E soprattutto ciò non significa che quella fortuna del dio "inattuale" fosse effettivamente inattuale ${ }^{35}$.

Il discorso di Jeanmaire si fonda su presupposti filologici e storici, e intende decostruire l'idea di un "dionisismo" come religione del vino e della possessione che avrebbe attraversato i secoli, senza subire variazioni. Tuttavia, Jesi sposta lo sguardo, modifica la posta in gioco dell'inattualità di Dioniso. Ciò che in Jeanmaire era, innanzitutto, una discussione da antichista, in Jesi diventa l'occasione di approfondire le riflessioni sul rapporto tra passato e presente, proponendo la sua idea dell'attualità del dionisiaco:

Del passato ciò che veramente importa è ciò che si dimentica. Ciò che si ricorda è soltanto sedimento e scoria. Ciò che importa, ciò che è destinato a sopravvivere, sopravvive apparentemente in segreto, in realtà nel modo più palese, giacché 
sopravvive come materia esistente di chi ha sperimentato il passato: come presente vivente, non come memoria di passato morto. L'esperienza dionisiaca consentiva, appunto, di teologizzare queste proposizioni. Dioniso era il dio del dolore poiché è dolorosa la perdita del passato quando il passato non è ricordato in quanto è rimasto presente. La meccanica e superficiale interpretazione dello schema di morte e rinascita intravisto nelle testimonianze della religiosità dionisiaca può essere modificata in questo senso: così come nell'iniziazione primordiale, l'esperienza di morte e rinascita è innanzitutto cambiamento, passaggio da uno stato ad un altro, da un tempo ad un altro. La morte che prelude la rinascita è abbandono del passato, il quale cessa di essere tale e non è ricordato poiché è divenuto presente. La rinascita è, appunto, l'esperienza di quel presente che comprende in sé tutto ciò che del passato era vivo ed è vivo: tutto ciò che non si ricorda ${ }^{36}$.

17 L'originalità di Jesi sta nell'abbandonare le teorie sul dio sofferente, sul dio che muore $\mathrm{e}$ risorge ${ }^{37}$. Lo studioso mette, invece, l'accento sulla dimensione del cambiamento e del passaggio di stato: la morte e la rinascita di Dioniso ricordano lo statuto del passato che rimane attivo nel presente. In questa prospettiva, Jesi riconosce le ragioni di Jeanmaire: non si può pensare che il dionisiaco di Lorenzo de' Medici e quello di Nietzsche assomiglino al "dionisismo originario". Tuttavia, in quelle interpretazioni e in quei fenomeni agiva "ciò che del passato si dimentica", le scorie dimenticate, ma perfettamente integrate al presente ${ }^{38}$. L'inattualità di Dioniso diviene allora pregnante per il nostro mondo contemporaneo: "l'esperienza religiosa dionisiaca è stata dimenticata, e dunque è divenuta materia vivente dei singoli presenti"39. Questa inattualità è un modo per interrogare il rapporto della cultura occidentale con il passato greco, di discutere il rapporto tra "Noi e i Greci" per utilizzare una formula che ha dato il titolo a un libro di Marcel Detienne ${ }^{40}$.

\section{Conclusione}

18 Negli anni di attività di Jesi, l'influente "inattualità" di Dioniso appariva prepotentemente negli ambiti più diversi della cultura. Il dio del vino, dell'estasi e delle donne era chiamato a partecipare, più o meno esplicitamente, ai grandi cambiamenti culturali e sociali che avevano animato la cultura occidentale dalla fine degli anni Sessanta: tracce della "presenza vivente" del dionisiaco, per dirla con Jesi, erano evidenti nei più rilevanti fenomeni culturali dell'epoca, dal Sessantotto al femminismo, dalla musica rock agli studi di genere ${ }^{41}$. Le pagine di Jesi affrontano alcuni aspetti specifici del dionisiaco che permettono di comprendere meglio la sua concezione dell'Antichità, del mito, della religione greca.

Due anni dopo aver scritto la prefazione al volume di Jeanmaire, nel 1974, Jesi pubblica un altro testo, forse meno conosciuto, nondimeno di grande interesse per il suo valore programmatico. È il manuale per la "prima classe del Liceo classico", intitolato La Vera Terra. Antologia di storici e altri prosatori greci sul mito e la storia ${ }^{42}$. L'antologia contiene anche una prestigiosa prefazione di Georges Dumézil dal titolo "Mito e storia. Appunti di un comparatista" dove il celebre professore del Collège de France loda il lavoro di Jesi, prova della risonanza internazionale del suo lavoro. Nell'introduzione, Jesi sottolinea la necessità dello studio della mitologia per la conoscenza della cultura antica:

[...] rispetto ad un apprezzamento non storico, ma storicistico, degli eventi umani, e dinanzi alla interpretazione di codesti eventi in termini di sociologismo volgare, la 
scienza del mito (non la manipolazione del mito) può offrire uno spazio di maggiore

rigore speculativo e al tempo stesso di maggiore libertà intellettuale ${ }^{43}$.

La "libertà intellettuale" è quella che il dionisiaco aveva promosso anche in anni recenti, attraverso le sue evoluzioni e permanenze nel tempo presente. Ma era anche l'occasione per esprimere una forte e chiara denuncia contro quello che era successo e stava ancora succedendo - in Grecia con la dittatura dei colonnelli. Ancora una volta Jesi mostrava il suo impegno politico e la sua capacità di associare lavoro intellettuale e impegno civile, in questo caso a difesa di una Grecia divenuta tristemente dal 21 aprile 1967 "terra chiusa e vietata" 44

PISI 2003: P. Pisi, «Dioniso da Nietzsche a Kerényi», Studi e Materiali di Storia delle Religioni 69, 1

(2003), 129-218.

\section{BIBLIOGRAFIA}

ANGELINI 1991: P. Angelini (a cura di), Cesare Pavese, Ernesto de Martino, La Collana Viola. Lettere 1945-1950, Torino 1991.

ARRIGO 2012: N. Arrigo, «Mito (simbolo) e Logos (allegoria) ne La terra e la morte e ne La luna e i falò di Cesare Pavese», Letteratura \& società 14, 2 (2012), 91-101.

BELTRAMETTI 2007: A. Beltrametti (a cura di), Studi e materiali per le Baccanti di Euripide. Storia Memorie Spettacoli, Pavia 2007.

BERNABÉ 2013: A. Bernabé, «Dionysos in the Mycenaean World», in A. Bernabé et al. (eds.), Redefining Dionysos, Berlin-Boston, 2013, 23-37.

BLOCH $1952^{2}$ : M. Bloch, Apologie pour l'histoire ou métier de l'historien, Paris $1952^{2}$ (prima ed. 1949). BRUHL 1953: A. Bruhl, Liber Pater. Origine et expansion du culte de Dionysos à Rome et dans le monde romain, Paris 1953.

CANCIK 1989: H. Cancik, Dioniso in Germania. Da Heinrich Heine a Walter F. Otto: una revisione di cent'anni, Roma 1989.

CHADWICK 1958: J. Chadwick, The Decipherment of Linear B, Cambridge 1958.

COMETA 2010: M. Cometa, «L'immagine in Jesi», in M. Belpoliti, E. Manera (a cura di), Furio Jesi, Milano 2010, 258-271.

COMPARINI 2017: A. Comparini, La poetica dei Dialoghi con Leucò di Cesare Pavese, Milano 2017.

CREUZER 1809: G. F. Creuzer, Dionysus, sive Commentationes academicae de rerum Bacchicarum orphicarumque originibus et caussis (sic), Heidelberg 1809.

CSAPO 2005: E. Csapo, Theories of Mythology, Oxford 2005.

DABDAD TRABULSI 1990: J. A. Dabdad Trabulsi, Dionysisme. Pouvoir et société en Grèce jusqu'à la fin de l'époque classique, Paris 1990, 24-31. 
DE MARTINO 1977: M. de Martino, La fine del mondo. Contributo all'analisi delle apocalissi culturali, Torino 1977.

DETIENNE 2007: M. Detienne, Noi e i Greci, Milano 2007 (ed. or. Paris 2005).

DoDDS 1940: E. R. Dodds, «Maenadism in the Bacchae», Harvard Theological Review 33 (1940), p. 155-176.

DODDS 1951: E. R. Dodds, The Greeks and the Irrational, Berkeley 1951.

EINAUDI 1983: Cinquant'anni di un editore. Le edizioni Einaudi negli anni 1933-1983. Breve iconografia, seguita dall'indice bibliografico degli autori collaboratori, dall'elenco delle collane, dagli indici per argomenti e per titoli, Torino, Einaudi, 1983.

FRANK 1994: M. Frank, Il dio a venire. Lezioni sulla Nuova Mitologia, trad. it., Torino 1994 (ed. or. Frankfurt 1982).

FRAZER 1911: J. G. Frazer, The Dying God, London 1911.

FUSILLO 2006: M. Fusillo, Il dio ibrido. Dioniso e le «Baccanti» nel Novecento, Bologna 2006.

GABRIELLI 2006: T. Gabrielli, «Sous le signe de Dionysos. Symbole, mythe et grécité chez Friedrich Creuzer», Archives de philosophie 69, 2 (2006), 243-261.

GALLAVOTTI, SACCONI 1961: C. Gallavotti, A. Sacconi, Inscriptiones Pyliae ad mycenaeam aetatem pertinentes, Roma 1961.

GRAF 1997: F. Graf, Il mito in Grecia, trad. it., Roma-Bari 1997 (ed. or. München-Zürich 1985).

HENRICHS 1984: A. Henrichs, «Loss of Self, Suffering, Violence: The Modern View of Dionysos from Nietzsche to Girard», Harvard Studies in Classical Philology 88 (1984), 205-240.

JEANMAIRE 1951: H. Jeanmaire, Dionysos. Histoire du culte de Bacchus, Paris 1951.

JEANMAIRE 1972: H. Jeanmaire, Dioniso. Religione e cultura in Grecia, trad. it. G. Glaesser, appendice e aggiornamenti biblibliografici di F. Jesi, Torino 1972.

LANG 1962: M. Lang, «The Palace of Nestor, Excavation of 1961», American Journal of Archaeology 66 (1962), 149-162.

JESI 1964: F. Jesi, «Cesare Pavese, il mito e la scienza del mito», Sigma 1 (1964), 95-120; ora in JESI 2002, 131-160.

JESI 1972a: F. Jesi, «Inattualità di Dioniso», in JEANMAIRE 1972, IX-XXIV. Ora in JESI 2001, 121-140.

JESI 1972b: F. Jesi, «Nuovi documenti e nuovi studi sulle origini di Dioniso», in JEANMAIRE 1972, 481-495.

JESI 1974: F. Jesi, La Vera Terra. Antologia di storici e altri prosatori greci sul mito e la storia, con un saggio di G. Dumézil, ad uso della prima classe del Liceo Classico, Torino 1974.

JESI 2001: F. Jesi, Materiali mitologici. Mito e antropologia nella cultura mitteleuropea, nuova edizione a cura di A. Cavalletti, Torino 2001.

JESI 2002: F. Jesi, Letteratura e mito, con un saggio di A. Cavalletti, Torino 2002.

JESI 2008: F. Jesi, Mito, nuova edizione con una nota di G. Schiavoni, Torino 2008 (prima ed. Milano 1973).

JESI, KERÉNYI 1999: F. Jesi, K. Kerényi, Demone e mito. Carteggio 1964-1968, a cura di M. Kerényi e A. Cavalletti, Macerata 1999. 
KERÉNYI 1951: K. Kerényi, «Un sacrificio dionisiaco», Dioniso 14 (1951), 139-156.

KERÉNYI 1992: K. Kerényi, Dioniso. Archetipo della vita indistruttibile, trad. it., Milano 1992 (ed. or. München - Wien 1976).

KIPPENBERG 2002: H. G. Kippenberg, La scoperta della storia delle religioni. Scienza delle religioni e modernità, trad. it., Brescia 2002

(ed. or. München 1997).

LANZA 1996: D. Lanza, «La tragedia e il tragico», in S. Settis (a cura di), I Greci. Storia Cultura Arte Società, vol. I: Noi e i Greci, Torino 1996, 469-505.

LANZA 2017: D. Lanza, Tempo senza tempo. La riflessione sul mito dal Settecento a oggi, Roma 2017.

LINCOLN 1999: B. Lincoln, Theorizing Myth. Narrative, Ideology, and Scholarship, Chicago 1999.

MCCUTCHEON 2000: R. McCutcheon, «Myth», in W. Braun, R. McCutcheon (eds.), Guide to the Study of Religion, London 2000, 190-208.

MASSA 2012: F. Massa, «The Meeting between Dionysus and the Christians in the Historiographical Debate of the XIX and XX Centuries», Historia Religionum 4 (2012), 159-182.

MANERA 2010: E. Manera, «Feste fiori sacrifici. Mito è nostalgia», in M. Belpoliti, E. Manera (a cura di), Furio Jesi, Milano 2010, 182-187.

MANERA 2015: E. Manera, «Memoria, violenza, scrittura: la "macchina mitologica" in Jesi», in G.

Leghissa, E. Manera (a cura di), Filosofie del mito nel Novecento, Roma 2015, 229-237.

MAUSS 1965: M. Mauss, Teoria generale della magia e altri saggi, Torino 1965.

MENCARINI 2013: B. Mencarini, L'inconsolabile Pavese, il mito e la memoria, Alessandria 2013.

MOREL 2015: Ph. Morel, Renaissance dionysiaque. Inspiration bachique, imaginaire du vin et de la vigne dans l'art européen (1430-1630), Paris 2015.

NALESINI 2013: O. Nalesini, «Onori e nefandezze di un esploratore. Note a margine a una recente biografia di Giuseppe Tucci», Annali dell'Università degli Studi di Napoli “L'Orientale” 73 (2013), 201-275.

PAVESE 1999: C. Pavese, Dialoghi con Leucò, introduzione di S. Givone, Torino 1999 (prima ed. 1947). PÉPIN 1958: J. Pépin, Mythe et allégorie. Les origines grecques et les contestations judéo-chrétiennes, Paris 1958.

ROHDE 2006: E. Rohde, Psiche. Culto delle anime e fede nell'immortalità presso i Greci, trad. it., Roma Bari 2006 (ed. or. Breisgau - Leipzig 1890-1894).

SMITH 1987: J. Z. Smith, s.v. «Dying and Rising Gods», Encyclopedia of Religion, New York 1987, vol. IV, 521-527.

SMITH 1990: J. Z. Smith, Drudgery Divine: On the Comparison of Early Christianities and the Religions of Late Antiquity, Chicago 1990.

SPINETO 1998: N. Spineto, «Tradizione e miti dionisiaci. Epifanie e ritorni del "Dio che viene"», Storiografia 2 (1998), 115-128.

SUARDI 2012: D. Suardi, «La religio mortis di Cesare Pavese», Doppiozero 12 novembre 2019. On line: https://www.doppiozero.com/dossier/cultura-di-destra/la-religio-mortis-di-cesare-pavese.

TARANTINO 2007: S. Tarantino, «La "Collana viola”: intervista a Gian Carlo Ferretti», La fabbrica del libro: bollettino di storia dell'editoria in Italia 13, 1 (2007), 39-42. 
VENTURI 2005: G. Venturi, «Cesare Pavese, Furio Jesi e il mito: una interpretazione», in M. Campanello (a cura di), Cesare Pavese: atti del convegno internazionale di studi, Torino, Santo Stefano Belbo, 24-27 ottobre 2001, Firenze 2005, 77-11.

\section{NOTE}

1. * Questo articolo si inserisce nell'ambito del progetto di ricerca sulla competizione religiosa nella tarda antichità da me diretto presso il Dipartimento di Storia dell'Università di Friburgo e finanziato dal Fondo Nazionale Svizzero per la ricerca scientifica (http://relab.hypotheses.org). PAVESE 1999, 73 (dal dialogo tra Eracle e Prometeo intitolato La rupe).

2. Si vedano JESI 1972a, JEANMAIRE 1951 e JEANMAIRE 1972.

3. JESI $1972 b$.

4. DODDS 1951. L'appendice era stata pubblicata come articolo una decina di anni prima: si veda DODDS 1940. Sull'importanza di Dodds nella storia degli studi si veda in particolare HENRICHS 1984, 224-230.

5. BRUHL 1953.

6. La stessa attenzione per le origini si ritrova anche negli studi relativi ad altre divinità del politeismo greco.

7. CREUZER 1809. Sull'idea della prima monografia dionisiaca, si veda CANCIK 1989, 24. Per l'origine egizia di Dioniso, si veda CREUZER 1809, IV, cap. I, par. 4.

8. ROHDE 1952, 267. Su Rohde, si veda CANCIK 1989, 40-51; sul suo rapporto con F. Nietzsche, si veda HENRICHS 1984, 224-226.

9. Da J. J. Bachofen a F. Nietzsche, i riferimenti al problema delle origini sono numerosi. Su questo tema, si veda LANZA 1996.

10. Si veda BLOCH $1952^{2}$, 5-9.

11. Sulla decifrazione della Lineare B si rimanda a CHADWICK 1958. Due sono le tavolette con il nome di Dioniso, trovate a Pilo: Xa 102 e Xb 1419. Si vedano GALLAVOTTI, SACCONI 1961 e LANG 1962. Sul ruolo di Dioniso nella religione micenea si vedano DABDAD TRABULSI 1990 e BERNABÉ 2013.

12. Si vedano KERÉNYI 1951 e KERÉNYI 1992. Sul Dioniso di Kerényi, si veda PISI 2003, 196-204.

13. Si veda EINAUDI 1983, 639.

14. EINAUDI 1983, 593. Sulla "Collana viola" si rimanda al carteggio tra Pavese e de Martino curato da ANGELINI 1991 e all'intervista a G. C. Ferretti in TARANTINO 2007.

15. MAUSS 1965 e De MARTINO 1977.

16. Un altro elemento interessante è quello relativo al traduttore italiano dell'opera, Gustavo Glaesser, che ha tradotto per Einaudi altri tre volumi: J. Jahn, Muntu. La civiltà africana moderna, prefazione E. de Martino, nel 1961; W. L. Shirer, Storia del Terzo Reich, nel 1962 e H. R. Hays, Dalla scimmia all'angelo. Due secoli di antropologia, nel 1974. In quegli anni, Glaesser traduce anche altre opere come il libro di S. Morenz, La religione egizia, pubblicato nel 1968 per Il Saggiatore e il Dizionario delle religioni di A. Bertholet, pubblicato nel 1964 da Editori Riuniti. Più interessante è che Glaesser era stato negli anni Trenta uno dei collaboratori dell'Istituto italiano per il Medio ed Estremo Oriente (IsMEO), fondato nel 1934 e che era stato l'autore di diversi articoli pubblicati nelle riviste Antieuropa. Rassegna mensile di azione e pensiero della giovinezza rivoluzionaria e Critica fascista. Sulle persone che collaborarono con l'IsMEO e sui rapporti con il fascismo si veda NALESINI 2013, in particolare 215.

17. Per uno studio sul ruolo di Dioniso nella letteratura e nell'arte del Rinascimento si veda MOREL 2015.

18. JESI 2008, 37-38. 
19. Sull'interpretazione del "mito" da parte di Jesi si vedano JESI 2008 e gli studi di VENTURI 2005, MANERA 2010 e MANERA 2015.

20. JESI 2001, 124.

21. Sul mito come allegoria nella tradizione classica si rimanda al lavoro classico di PéPIN 1958. Sulla storiografia moderna si vedano, tra i numerosi saggi che si potrebbero citare, LINCOLN 1999 e MCCUTCHEON 2000.

22. Per i giudizi critici sui Dialoghi con Leucò è utile l'«Antologia della critica» pubblicata in PAVESE 1999, 197-222 e MENCARINI 2013. Per una riflessione jesiana sul mito in Pavese si veda JESI 1964, ARRIGO 2012 e SUARDI 2012.

23. Così LANZA 2017, 150-151. Si veda anche JESI 2002, 133-134.

24. JESI 2001, 124-125.

25. JESI 2001, 137.

26. Ibid.

27. Sul Prometeo di Pavese si veda LANZA 2017, 148-150.

28. JESI 2001, 125.

29. Sulla storia degli studi dionisiaci di quest'epoca mi permetto di rimandare a MASSA 2012, 160-177.

30. Sulla "Nuova Mitologia" si veda lo studio di FRANK 1994 e KIPPENBERG 2002, 140-146.

31. Su questo aspetto si veda anche SPINETO 1998, 124-125.

32. La monografia su Dioniso anticipa l'opera fondamentale di Creuzer, Symbolik und Mythologie der alten Völker, pubblicata per la prima volta tra il 1810 e il 1812, in cui cercava di provare, attraverso l'analisi dell'uso delle formule liturgiche, la stretta dipendenza del cristianesimo dai misteri dionisiaci. Sul ruolo di Dioniso nell'opera di Creuzer si rimanda a GABRIELLI 2006.

33. La bibliografia sul Dioniso di Nietzsche è ovviamente molto ampia. In questa sede si rimanda soltanto a HENRICHS 1984, 206-223, particolarmente interessante per il suo punto di vista di studioso del mondo classico.

34. Il rapporto tra Jesi e Kerényi si era interrotto nel 1968, come ricorda P. Lanfranchi, nell'articolo pubblicato su questo numero di MYTHOS. A questo proposito, si veda JESI, KERÉNYI 1999 e COMETA 2010.

35. JESI 2001, 126.

36. JESI 2001, 126-127.

37. La definizione canonica del "dying and rising god si trova in FRAZER 1911. Su questa categoria si veda SMITH 1987 e SMITH 1990, 85-115.

38. JESI 2001, 128.

39. Ibid.

40. DETIENNE 2007.

41. Diversi saggi, con metodi e angolature differenti, hanno cercato di ricostruire le interpretazioni di Dioniso nel Novecento: mi limito a rimandare a FUSILLO 2006 e BELTRAMETTI 2007.

42. JESI 1974.

43. JESI 1974, XIX.

44. JESI 1974, XXII. 


\section{RIASSUNTI}

L'articolo propone una riflessione sul saggio "Inattualità di Dioniso" scritto da Furio Jesi come introduzione alla traduzione italiana del volume di Henri Jeanmaire, Dionysos. Histoire du culte de Bacchus, pubblicata da Einaudi nel 1972. L'obiettivo è di situare il testo di Jesi nel panorama degli studi su Dioniso della seconda metà del Novecento e di metterne in evidenza gli elementi di attualità. Si analizzeranno, in particolare, la concezione jesiana della categoria del "dionisiaco" e le tracce della sua "presenza vivente" nel dibattito intellettuale dell'epoca.

The article deals with the essay "Inattualità di Dioniso" written by Furio Jesi as an introduction to the Italian translation of the volume Dionysos. Histoire du culte de Bacchus, by Henri Jeanmaire (Einaudi, 1972). The article places and discusses Jesi's text in the context of studies on Dionysus of the second half of the twentieth century. The analysis focuses on the Jesian conception of the conception of "Dionysian" as a category still looming in the intellectual debate.

\section{INDICE}

Keywords : Jesi Furio, Dionysus, Dionysian, Jeanmaire Henri, Pavese Cesare

Parole chiave : Jesi Furio, Dioniso, dionisiaco, Jeanmaire Henri, Pavese Cesare

\section{AUTORE}

\section{FRANCESCO MASSA}

Université de Fribourg

Institut du monde antique et byzantin, 16 rue Pierre-Aeby, CH-1700 Fribourg (Suisse), francesco.massa(at)unifr.ch 